\title{
Predicting mortality in patients with sepsis in the emergency department by using combinations of biomarkers and clinical scoring systems: a systematic review.
}

Kirby Tong-Minh ( $\nabla$ k.tong-minh@erasmusmc.nl )

Erasmus MC https://orcid.org/0000-0002-8373-0011

Iris Welten

Erasmus MC

Henrik Endeman

Erasmus MC

Tjebbe Hagenaars

Erasmus MC

Christian Ramakers

Erasmus MC

Diederik Gommers

Erasmus MC

Eric van Gorp

Erasmus MC

Yuri van der Does

Erasmus MC

\section{Research}

Keywords: Sepsis, biomarkers, emergency department, prediction model

Posted Date: October 7th, 2020

DOI: https://doi.org/10.21203/rs.3.rs-86239/v1

License: (c) This work is licensed under a Creative Commons Attribution 4.0 International License. Read Full License 


\section{Abstract}

Introduction Sepsis can be detected in an early stage in the emergency department (ED) by biomarkers and clinical scoring systems. A combination of multiple biomarkers or biomarker with clinical scoring system might result in a higher predictive value on mortality. The goal of this systematic review is to evaluate the available literature on combinations of biomarkers and clinical scoring systems on 1-month mortality in patients with sepsis in the ED.

Methods We performed a systematic search using MEDLINE, PubMed, EMBASE and Google Scholar. Articles were included if they evaluated at least one biomarker combined with another biomarker or clinical scoring system and reported the diagnostic accuracy on 28 or 30 day mortality by area under the curve (AUC) in patients with sepsis.

Results We found 18 articles in this systematic review. In these 18 articles, a total of 35 combinations of biomarkers and clinical scoring systems were studied of which 33 unique combinations. In total, seven different clinical scoring systems and 21 different biomarkers were investigated. The combination of procalcitonin (PCT), lactate, interleukin-6 (IL-6) and Simplified Acute Physiology Score-2 (SAPS-2) resulted in the highest AUC on 1-month mortality.

Conclusion In this systematic review, the combination of PCT, IL-6, lactate and the SAPS-2 score had the highest AUC on 1month mortality in patients with sepsis in the ED. The studies we found in this review were too heterogeneous to conclude that a certain combination it should be used in the ED to predict 1-month mortality in patients with sepsis.

\section{Introduction}

Sepsis is a life threatening condition, and is the leading cause of in-hospital mortality in Europe. ${ }^{1}$ Early detection of sepsis is essential to timely start appropriate treatment. ${ }^{2,3}$ Early stage sepsis, in patients with a suspected infection, is often undiagnosed, causing a delay in treatment and increased mortality. ${ }^{4} 5$ The emergency department (ED) is often the first setting during hospital stay where patients with a suspected infection are systematically evaluated, where early stages of sepsis can be detected. There is however, a limited timeframe in the ED in which decisions about treatment and patient disposition must be made.

Multiple organ systems and pathways are involved in the pathophysiology of sepsis. ${ }^{6}$ After a microorganism infects the body, multiple immune responses are activated. Different immune cells are activated, which express a series of membrane receptors, endothelial and tissue factors are released, and the complement system is activated. In sepsis, this immune response is dysregulated and excessive, ultimately resulting in multi-organ failure. ${ }^{7}$ This response involves dysregulation by both hyperinflammation and immune suppression. In these different stages of sepsis different cytokines, peptides and other signaling molecules are elevated and can be detected in the bloodstream as biomarkers.

Clinical scoring systems, which are often used to detect sepsis, rely on vital parameters. However, when vital parameters are abnormal, the patient might already be in an advanced stage of sepsis. Using biomarkers to detect sepsis, early stages of sepsis could be detected before vital signs turn abnormal. Furthermore, adding biomarkers to clinical scoring systems might increase predictive power of these systems. A large variety of biomarkers in patients with sepsis have been studied. Pierrakos et al. reviewed the literature on biomarkers in sepsis in 2010 and found that there are over 100 different biomarkers studied, of which none have made it to clinical practice except C-reactive protein (CRP) and procalcitonin (PCT). ${ }^{8}$ This study concluded that a combination of several biomarkers may be more effective. Several studies support this claim and show that combining biomarkers with clinical scoring systems or combining multiple biomarkers result in a more accurate prediction of mortality in patients with infectious diseases in the ED. ${ }^{9,10}$

With the large number of biomarkers already studied, the potential number of combinations of biomarkers and clinical scoring systems is even greater. 
The goal of this review is to systematically assess the available literature on combinations of biomarkers and clinical scorings systems in patients with sepsis in the ED to predict 1-month mortality.

\section{Objectives}

What combination of biomarkers and clinical scoring system has the highest area under the curve (AUC) to predict 1-month mortality in patients with sepsis in the ED?

\section{Methods}

We conducted this systematic review following the Preferred Reporting Items for Systematic Reviews and Meta-analyses (PRISMA) guidelines. ${ }^{11}$ The study was registered on PROSPERO, the register for systematic reviews under reference number 165580.

\section{Literature search}

We performed a systematic search of literature by an information specialist using MEDLINE, PubMed, EMBASE and Google Scholar. The search included articles in English published up to June 2020. The search included sepsis in combination with biomarkers in the ED. The full search strategy can be found in the Appendix.

\section{Outcome definitions}

Biomarkers were considered as any laboratory blood test performed in the ED. A clinical scoring system was defined as any scoring system using a combination of patient characteristic with or without laboratory testing used for prognostic purpose. We used 1-month mortality as outcome, which was defined as either 28 or 30-day mortality.

\section{Study selection}

After the initial search, studies were screened by title and abstract by two independent reviewers. The results were compared and discrepancies were resolved by discussion. If no consensus was achieved, a third reviewer acted as referee. The remaining studies were screened on inclusion and exclusion criteria using the full text (figure 1).

\section{Selection criteria}

During title and abstract screening, articles were included if they evaluated at least one biomarker or clinical scoring system in any infectious disease. During full text screening, articles were included if they evaluated at least one biomarker combined with another biomarker or clinical scoring system and reported the diagnostic accuracy on 28 or 30 day mortality by AUC in patients with sepsis.

Studies in children and studies of which the full text was not available in English were excluded.

\section{Data collection and quality assessment}

Data was extracted in a predefined spreadsheet, which included the biomarkers and clinical scoring system used, AUC of the combination of biomarkers and clinical scoring system, age, inclusion and exclusion criteria and moment of blood collection . The quality of each study was assessed using Quality Assessment of Diagnostic Accuracy Studies (QUADAS2). ${ }^{12}$ This risk of bias was assessed during the QUADAS-2 assessment and reported when bias was suspected.

\section{Results}

We found 5826 articles after conducting our search in PubMed, EMBASE, Medline Ovid, Web of Science, Cochrane Central and Google Scholar. After removal of duplicates, 3771 articles remained. These articles were screened on title and abstract, 
after which 83 articles were included for full text screening. After full text screening, 65 articles were excluded. This resulted in 18 articles included for final data synthesis. (figure 1)

In the 18 articles that were included in this systematic review, a total of 35 combinations of biomarkers and clinical scoring systems were studied of which 33 unique combinations. In total, seven different clinical scoring systems and 21 different biomarkers were investigated. (table 1)

The Mortality in Emergency Department Sepsis (MEDS) score was the most used clinical scoring system, which was studied in 9 articles. The second most commonly used scoring system was the Acute Physiology and Chronic Health Evaluation II (APACHE II) score, which was studied in 6 articles. The most commonly studied biomarker was PCT, which was studied in 7 articles. The combination of PCT with the MEDS score was the most studied combination of biomarker and clinical score and was studied in 3 different articles. There were no other combinations of biomarkers that were used by more than a single article.

The number of patients included in the studies ranged from 114 to 1318. The AUC of the combinations of biomarkers and clinical scoring systems ranged from 0,690 to 0,939 . The highest AUC was achieved by the combination of Simplified Acute Physiology Score (SAPS II), PCT, lactate and interleukine-6 (IL-6), which yielded an AUC of 0,939 by Viallon et al. ${ }^{13}$, followed by the combination of SAPS-2 and soluble urokinase-type plasminogen activator receptor (suPAR) with an AUC 0.930 by Kofoed et al. ${ }^{14}$

Different inclusion criteria were used to classify patients as having sepsis. The most common criteria used were two SIRS criteria in combination with an infection, used by eight articles. ${ }^{13-20}$ The second most used inclusion criterion was the 2001 International Sepsis Definitions ${ }^{21}$, which was used by seven articles. ${ }^{17,22-27}$ One article used the sampling of blood cultures as inclusion criterion. ${ }^{28}$ One article included patients with symptoms of systemic infection in which PCT or blood cultures were taken within 24 hours of admission. ${ }^{29}$ One article used the Sepsis-3 definition ${ }^{7}$ as inclusion criterion. ${ }^{30}$

\section{QUADAS-2 quality assessment}

The quality assessment using the QUADAS-2 criteria can be found in table 2 . Three studies were at risk of bias. ${ }^{15,22,31}$ These studies used multiple exclusion criteria, enrolling a very selected group of patients with sepsis. The exclusion criteria were mostly patients who were immunocompromised, for example due to immunosuppressant medication, malignancies or autoimmune diseases. ${ }^{22,31}$

Only a minority of the articles used a predefined cutoff of the studied biomarkers and scoring system. ${ }^{17,25,27,29}$

There were no concerns of possible bias in flow and timing of the biomarkers and scoring systems that were investigated. Blood sampling was performed during ED visit in all studies.

The studied population matched the review question in all but two studies. Nino et al. ${ }^{27}$ also included ICU patients and Koefoed et al. ${ }^{14}$ also included patients from a general ward along with ED patients. These studies were included nonetheless, because blood sampling occurred during admission. 


\begin{tabular}{|c|c|c|c|c|c|c|c|}
\hline 1or, year & $\begin{array}{c}\text { Number of } \\
\text { patients }\end{array}$ & Biomarker 1 & $\begin{array}{c}\text { Biomarker } \\
2 \\
\end{array}$ & $\begin{array}{c}\text { Biomarker } \\
3 \\
\end{array}$ & $\begin{array}{c}\text { Clinical score } \\
1 \\
\end{array}$ & $\begin{array}{c}\text { Clinical score } \\
2 \\
\end{array}$ & AUC \\
\hline \multirow[t]{2}{*}{$2019^{29}$} & 1318 & PCT & & & qSOFA & & 0,73 \\
\hline & & $\mathrm{CRP}$ & & & qSOFA & & 0,69 \\
\hline $\begin{array}{l}\text { amoto, } \\
j^{28}\end{array}$ & 1262 & CRP & & & CURB65 & & 0,77 \\
\hline \multirow[t]{2}{*}{$2013^{26}$} & 859 & Presepsin & & & MEDS & & 0,731 \\
\hline & & Presepsin & & & APACHE-2 & & 0,734 \\
\hline \multirow[t]{3}{*}{$\lg , 2014^{24}$} & 680 & Copeptin & & & MEDS & & 0,851 \\
\hline & & Cortisol & & & MEDS & & 0,833 \\
\hline & & PCT & Cortisol & Copeptin & MEDS & & 0,891 \\
\hline \multirow[t]{3}{*}{ a, $2014^{25}$} & 680 & Lactate & & & MEDS & & 0,81 \\
\hline & & Lactate & & & APACHE-2 & & 0,81 \\
\hline & & Lactate & & & SOFA & & 0,82 \\
\hline $2013^{18}$ & 680 & sTM & & & MEDS & & 0,805 \\
\hline \multirow[t]{3}{*}{$2018^{31}$} & 655 & PCT & sPD-1 & & MEDS & & 0,843 \\
\hline & & PCT & & & MEDS & & 0,792 \\
\hline & & sPD-1 & & & MEDS & & 0,829 \\
\hline ), $2017^{27}$ & 563 & TIMP1 & MMP9 & & CHARLSON & SOFA & 0,838 \\
\hline $2013^{19}$ & 501 & PCT & & & MEDS & & 0,813 \\
\hline \multirow[t]{3}{*}{$\lg , 2016^{22}$} & 480 & $\operatorname{Ig} E$ & & & APACHE-2 & & 0,8 \\
\hline & & $\operatorname{Ig} E$ & & & SOFA & & 0,781 \\
\hline & & $\operatorname{Ig} E$ & & & MEDS & & 0,89 \\
\hline \multirow[t]{3}{*}{$g, 2014^{23}$} & 480 & NGAL & & & MEDS & & 0,858 \\
\hline & & TIMP-1 & & & MEDS & & 0,882 \\
\hline & & PCT & & & MEDS & & 0,782 \\
\hline ning, & & Angiopoientin- & & & & & \\
\hline $3^{20}$ & 314 & 2 & IL-6 & & & & 0,72 \\
\hline \multirow[t]{4}{*}{ a, $2014^{17}$} & 295 & H-FABP & & & MEDS & & 0,853 \\
\hline & & H-FABP & & & APACHE-2 & & 0,826 \\
\hline & & Troponine-I & & & APACHE-2 & & 0,811 \\
\hline & & Troponine-I & & & MEDS & & 0,825 \\
\hline \multicolumn{8}{|l|}{ lessis, } \\
\hline \multirow[t]{2}{*}{$3^{15}$} & 203 & Nucleosomes & & & APACHE-2 & & 0,84 \\
\hline & & Cell free DNA & & & APACHE-2 & & 0,81 \\
\hline \multirow[t]{2}{*}{ ed, $2008^{14}$} & 161 & suPAR & sTREM-1 & & SAPS-2 & & 0,89 \\
\hline & & suPAR & & & SAPS-2 & & 0,93 \\
\hline on, $2008^{13}$ & 131 & PCT & IL-6 & Lactate & SAPS-2 & & 0,939 \\
\hline io, $2015^{16}$ & 114 & Presepsin & & & MEDS & & 0,878 \\
\hline y, $2019^{30}$ & 113 & IL-6 & PTX3 & & & & 0,637 \\
\hline
\end{tabular}

Table 1: Biomarkers. PCT: Procalcitonin, CRP: C-reactive protein, sTM: soluble thrombomodulin, sPD-1: soluble programmed death 1, TIMP-1: Tissue inhibitor of metalloproteinase-1, MMP9: Matrix metallopeptidase 9, NGAL: Neutrophil gelatinase-associated lipocalin, IL-6: interleukin-6, H-FABT: heart fatty acid binding protein, suPAR: soluble urokinase-type plasminogen activator receptor, PTX3: Pentraxin 3

Scoring systems. qSOFA: quick Sequential Organ Failure Assessment, MEDS: Mortality in Emergency Department Sepsis, APACHE-2: Acute Physiologic Assessment and Chronic Health Evaluation II, SOFA: Sequential Organ Failure Assessment, SAPS-2: Simplified Acute Physiology Score 2, CHARLSON 


\begin{tabular}{|c|c|c|c|c|c|c|}
\hline \multirow[b]{2}{*}{ Author } & \multicolumn{3}{|l|}{ Risk of bias } & \multicolumn{3}{|c|}{ Applicability } \\
\hline & $\begin{array}{l}\text { Patient } \\
\text { selection }\end{array}$ & Index test & $\begin{array}{l}\text { Flow and } \\
\text { timing }\end{array}$ & $\begin{array}{l}\text { Patient } \\
\text { selection }\end{array}$ & Index test & $\begin{array}{l}\text { Reference } \\
\text { standard }\end{array}$ \\
\hline $\begin{array}{l}\text { Yu, } 2019^{29} \\
\text { Yamamoto }\end{array}$ & (2) & (:) & ;) & (2) & (:) & NA \\
\hline $2015^{2 B}$ & (:) & (:) & (:) & ;) & (-) & NA \\
\hline Liu, $2013^{26}$ & (:) & (:) & (:) & ;) & (-): & NA \\
\hline Zhang, $2014^{24}$ & (:) & (:) & (:) & (:) & (:) & NA \\
\hline Chen, $2014^{25}$ & (-): & (:) & (:) & (:) & (-): & NA \\
\hline Yin, $2013^{18}$ & ;) & (:) & (:) & (:) & (:) & NA \\
\hline Zhao, $2018^{31}$ & (:) & (:) & (:) & (:) & (:) & NA \\
\hline Niño, $2017^{27}$ & (:) & (:) & :) & (1) & (:) & NA \\
\hline Zhao, $2013^{19}$ & (:) & (:) & (:) & (:) & (:) & NA \\
\hline Zhang, $2016^{22}$ & (2) & (:) & (-) & (:) & (-) & NA \\
\hline Wang, 2014 & (:) & (:) & (:) & 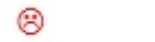 & (-): & NA \\
\hline Henning, $2019^{20}$ & (-) & (:) & (:) & (:) & (-) & NA \\
\hline Chen, $2014^{17}$ & :) & (:) & (:) & (:) & (:) & NA \\
\hline Duplessis, $2018^{15}$ & (2) & (:) & (:) & (:) & (:) & NA \\
\hline Kofoed, $2008^{14}$ & (:) & (:) & (:) & (:) & (:) & NA \\
\hline Viallon, $2008^{13}$ & (:) & (:) & (:) & (:) & (-) & NA \\
\hline Carpio, $2015^{16}$ & (:) & (:) & (:) & (:) & (-): & NA \\
\hline Song, $2019^{30}$ & (-): & (:) & (:) & (:) & (:): & NA \\
\hline
\end{tabular}

\section{Discussion}

Biomarkers and clinical scoring systems help physicians to detect sepsis in an early stage in the ED. In this systematic review we investigated the combinations of biomarkers and clinical scoring systems to predict 1-month mortality in patients with sepsis. We found 18 different studies in which 33 combinations of biomarkers and clinical scoring system were investigated. The combination of PCT, lactate, IL-6 and SAPS-2 resulted in the highest AUC on 1-month mortality. ${ }^{13}$ Despite the high AUC found in this study, this specific combination has not been adopted in the latest guidelines for surviving sepsis. ${ }^{2}$ The SAPS-2 score is a clinical scoring system, using four vital parameters, seven laboratory tests and four other patient characteristics and was originally developed for patients in the ICU or general wards to predict in-hospital mortality. Combining this clinical scoring system with another three biomarkers results in a total of 18 variables used to predict 30day mortality in this study. This study enrolled 131 subjects, of which 19 died. Therefore, the high AUC found in this study may possibly be due to overfitting by using too many predictors in the multivariate logistic regression analysis. ${ }^{32,33}$

Lactate is a product of anaerobic glycolysis and is often elevated in patients with sepsis. It has been adopted as criterion for septic shock in the Sepsis-3 definitions. ${ }^{7}$ We found two studies using lactate in combination with other biomarkers or clinical scoring system, both with a high predictive value on 1-month mortality. ${ }^{13,17}$ Unlike many novel biomarkers, lactate is widely available as a standard measurement during the workup in the ED. Therefore, lactate is an important biomarker in assessing the severity of sepsis in the ED. IL- 6 is an inflammatory cytokine and plays an important role in the early phase of sepsis. ${ }^{34}$ However, the prognostic values of IL- 6 are controversial due to the short window in which IL- 6 rises and falls during inflammation and infection. ${ }^{35}$

The SAPS-2 score was also used in combination with suPAR by Kofoed et al. ${ }^{14}$, resulting in an AUC of 0.930 , which was the second highest AUC we found in our study. These findings suggest that the SAPS-2 is a clinical scoring system with a high diagnostic accuracy on 30-day mortality, although it has not been validated for assessing severity of disease in the ED. 
However, the limitation of an overfitted prediction model in a relatively small cohort is also present in the study of Kofoed et al., with only 161 patients enrolled of which 9 patients died. suPAR is a biomarker which has been investigated as general disease severity biomarker, mostly in the ED. A large study showed that suPAR is an accurate predictor of mortality, but does not influence disposition or clinical outcome when it was used in the ED. ${ }^{36}$ In a meta-analysis, suPAR showed similar results as PCT in diagnosing sepsis. ${ }^{37}$

We found four studies using PCT combined with another biomarker or clinical scoring system. PCT has been studied as biomarker for bacterial infections and disease severity in infectious diseases. PCT is the precursor of calcitonin and physiologically produced by thyroid cells. In bacterial infections it is also synthesized outside of the thyroid, and rises rapidly in systemic infections. It is often referred to as the biomarker with most potential of replacing or substituting CRP. ${ }^{38}$ However, PCT has yet to establish a role in routine care in the ED. ${ }^{39}$ Combining PCT with other biomarkers or clinical scoring systems, we found an increase in predictive value on 30-day mortality. From all available sepsis biomarkers, PCT is probably the most well-known among physicians in the ED. Combining PCT with clinical scoring systems, as done in many studies, might therefore be the key in being adopted as part of regular care.

The MEDS score was the most used clinical scoring system used in combination with biomarkers. The MEDS score is a risk prediction score specifically for patients with suspected sepsis in the ED. ${ }^{40}$ It consists of nine items which can easily be scored in the ED setting and results in a total score, categorized in 5 groups, which corresponds to a certain mortality risk. The AUC of the MEDS score combined with different biomarkers ranged from 0.731 to 0.891 , indicating a moderate to good predictive value on 30-day mortality. Other clinical scoring systems we found in combination with biomarkers were the APACHE-2 and SAPS-2 score. These clinical scoring systems are mainly developed for use in the ICU and general wards. Despite being accurate predictors of disease severity, these clinical scoring systems may be less feasible for use in the ED, due to their complexity and large number of clinical parameters needed. In a prospective study comparing different clinical scoring systems individually in the ED, the MEDS score resulted in an AUC of 0.94 on 30-day mortality, which was higher than the SOFA or PIRO score. ${ }^{41}$ However, another study which also compares different clinical scoring systems concluded that the APACHE-2 score is superior to the MEDS and SOFA score. ${ }^{42}$

Three studies investigated biomarkers which are otherwise known as hormones and other functional circulating peptides, including IgE, cortisol, cell free DNA and nucleosomes. ${ }^{15,22,24}$ Zhang et al. ${ }^{22}$ studied IgE in combination with the MEDS score and found that adding IgE to the MEDS score resulted in a higher AUC than the MEDS score alone. This study emphasizes the multifactorial entity of sepsis, hypothesizing that IgE either plays a role in general immune activation during sepsis or is a marker of cytokine regulation/dysregulation. Another study by Zhang et al. ${ }^{24}$ investigated hormones and biomarkers from the hypothalamic-pituitary-adrenal axis and showed that cortisol and copeptin are associated with 30day mortality and that combining these biomarkers with the MEDS score resulted in added value over using each biomarker individually. Cortisol has been identified as essential hormone in the immune response in sepsis and elevated levels of cortisol are associated with severity of sepsis. ${ }^{43}$ Extracellular cell free DNA and nucleosomes, basic units of DNA packaging, reflect cellular apoptosis and are therefore tested as predictors of severity of sepsis in the study of Duplessis et al. ${ }^{15}$ In this study the authors show that adding nucleosomes to the APACHE-2 score improved the AUC on predicting mortality. Adding cell free DNA to the APACHE-2 score did not result in a better predictive value. These studies emphasize that biomarkers originating from different pathways in sepsis can be used as predictor of disease severity.

\section{Limitations}

Our study has several limitations. We included articles that investigated the predictive value of biomarkers combined with clinical scoring systems on disease severity in sepsis. We used 1-month mortality as endpoint for severity of sepsis. However, there are many more biomarkers that have been investigated using other endpoints as marker of disease severity of sepsis. These endpoints to define severity of sepsis range from ICU admission to long term mortality. Despite the fact that these endpoints also are a surrogate marker of disease severity, these articles were not included because a comparison 
of these endpoints would not be possible. An example of a commonly used scoring system not included in this study is the National Early Warning System (NEWS). The NEWS score is widely adopted as early warning score to identify patients at risk of clinical deterioration both in the ED and the hospital wards, where it also has been shown to be superior to the qSOFA score. ${ }^{44}$ Adding biomarkers to the NEWS score could improve its predictive value even further. However, no studies on our specific endpoint with the NEWS score and biomarkers were found.

The definition of sepsis has changed over time, which is also reflected by the different inclusion criteria used by the studies we found. Most studies used the sepsis criteria as defined in $2001^{21}$, and only a few studies used the latest Sepsis-3 criteria. ${ }^{7}$ Other studies used two or more SIRS criteria in combination with an infection, but there were also studies that included patients with only blood cultures taken. This might have resulted in a heterogeneous groups of patient populations in which the severity of sepsis in the ED differs at the moment of inclusion. Some studies included patients with already diagnosed sepsis, while other studies also included patients at risk or suspected of sepsis.

All but three studies did not use predefined cut-off values for the biomarkers. It is common practice not to do so as an AUC is usually constructed using biomarkers and scoring systems on a continuous scale. However, for translating these prediction models to clinical practice, using a predefined cutoff, categorizing the biomarker or clinical scoring system in a high or low risk category, is preferred.

The study population size of the included studies ranged from 114 to 1318. In these relatively small sample sizes, overfitting of prediction models often is a problem. When there are less than 10 fatal cases per predictor, the risk of overfitting of prediction models is high, resulting in an unrealistically high AUC. ${ }^{33}$ When also including clinical scoring systems, this problem is even bigger, since the clinical scoring system already consists of multiple predictors.

Conducting a meta-analysis to compare the outcomes of different studies would be preferred, but was not feasible. The variety in biomarkers and clinical scoring systems used was too large to compare one to another.

\section{Conclusion}

In this systematic review, the combination of PCT, IL-6, lactate and the SAPS-2 score had the highest AUC on 1-month mortality in patients with sepsis in the ED. This finding should be validated in an external cohort as this AUC may be overfitted. The studies we found in this review were too heterogeneous to conclude that a certain combination it should be used in the ED to predict 1-month mortality in patients with sepsis. Other potential biomarkers for risk stratification in patients with sepsis in the ED include PCT and the MEDS score, and it is interesting to further investigate if these can be used in clinical practice for decision making.

\section{Declarations}

Ethics approval and consent to participate

Not applicable.

\section{Consent for publication}

Not applicable.

\section{Availability of data and materials}

The datasets used and/or analysed during the current study are available from the corresponding author on reasonable request. 
The authors declare that they have no competing interests.

\section{Funding}

This manuscript was not funded by any funding body.

\section{Authors' contributions}

KTM, IW, and YD were involved in the conception or design of the manuscript. KTM and IW did the screening of the articles and YD acted as referee. KTM and YD did the analysis and interpretation of the data. KTM drafted the manuscript. KTM, IW, $H E, T H, C R, D G, E G$ and YD were involved in the critical revision of the manuscript and final approval of the manuscript.

\section{Acknowledgements}

The authors wish to thank Wichor Bramer and Sabrina Meertens-Gunput from the Erasmus MC Medical Library for developing and updating the search strategies.

\section{References}

1. Vincent JL, Jones G, David S, Olariu E, Cadwell KK. Frequency and mortality of septic shock in Europe and North America: a systematic review and meta-analysis. Crit Care. 2019;23(1):196.

2. De Backer D, Dorman T. Surviving Sepsis Guidelines: A Continuous Move Toward Better Care of Patients With Sepsis. JAMA. 2017;317(8):807-8.

3. Rivers E, Nguyen B, Havstad S, Ressler J, Muzzin A, Knoblich B, et al. Early goal-directed therapy in the treatment of severe sepsis and septic shock. N Engl J Med. 2001;345(19):1368-77.

4. Hayden GE, Tuuri RE, Scott R, Losek JD, Blackshaw AM, Schoenling AJ, et al. Triage sepsis alert and sepsis protocol lower times to fluids and antibiotics in the ED. Am J Emerg Med. 2016;34(1):1-9.

5. Husabo G, Nilsen RM, Flaatten H, Solligard E, Frich JC, Bondevik GT, et al. Early diagnosis of sepsis in emergency departments, time to treatment, and association with mortality: An observational study. PLoS One. 2020;15(1):e0227652.

6. Gyawali B, Ramakrishna K, Dhamoon AS. Sepsis: The evolution in definition, pathophysiology, and management. SAGE Open Med. 2019;7:2050312119835043.

7. Singer M, Deutschman CS, Seymour CW, Shankar-Hari M, Annane D, Bauer M, et al. The Third International Consensus Definitions for Sepsis and Septic Shock (Sepsis-3). JAMA. 2016;315(8):801-10.

8. Pierrakos C, Vincent JL. Sepsis biomarkers: a review. Crit Care. 2010;14(1):R15.

9. Schuetz P, Hausfater P, Amin D, Amin A, Haubitz S, Faessler L, et al. Biomarkers from distinct biological pathways improve early risk stratification in medical emergency patients: the multinational, prospective, observational TRIAGE study. Crit Care. 2015;19:377.

10. Travaglino F, De Berardinis B, Magrini L, Bongiovanni C, Candelli M, Silveri NG, et al. Utility of Procalcitonin (PCT) and Mid regional pro-Adrenomedullin (MR-proADM) in risk stratification of critically ill febrile patients in Emergency Department (ED). A comparison with APACHE II score. BMC Infect Dis. 2012;12:184.

11. Moher D, Shamseer L, Clarke M, Ghersi D, Liberati A, Petticrew M, et al. Preferred reporting items for systematic review and meta-analysis protocols (PRISMA-P) 2015 statement. Syst Rev. 2015;4:1.

12. Whiting PF, Rutjes AW, Westwood ME, Mallett S, Deeks JJ, Reitsma JB, et al. QUADAS-2: a revised tool for the quality assessment of diagnostic accuracy studies. Ann Intern Med. 2011;155(8):529-36.

13. Viallon A, Guyomarc'h S, Marjollet O, Berger C, Carricajo A, Robert F, et al. Can emergency physicians identify a high mortality subgroup of patients with sepsis: role of procalcitonin. Eur J Emerg Med. 2008;15(1):26-33. 
14. Kofoed K, Eugen-Olsen J, Petersen J, Larsen K, Andersen O. Predicting mortality in patients with systemic inflammatory response syndrome: an evaluation of two prognostic models, two soluble receptors, and a macrophage migration inhibitory factor. Eur J Clin Microbiol Infect Dis. 2008;27(5):375-83.

15. Duplessis C, Gregory M, Frey K, Bell M, Truong L, Schully K, et al. Evaluating the discriminating capacity of cell death (apoptotic) biomarkers in sepsis. J Intensive Care. 2018;6:72.

16. Carpio R, Zapata J, Spanuth E, Hess G. Utility of presepsin (SCD14-ST) as a diagnostic and prognostic marker of sepsis in the emergency department. Clin Chim Acta. 2015;450:169-75.

17. Chen YX, Li CS. The prognostic and risk-stratified value of heart-type fatty acid-binding protein in septic patients in the emergency department. J Crit Care. 2014;29(4):512-6.

18. Yin Q, Liu B, Chen Y, Zhao Y, Li C. The role of soluble thrombomodulin in the risk stratification and prognosis evaluation of septic patients in the emergency department. Thromb Res. 2013;132(4):471-6.

19. Zhao Y, Li C, Jia Y. Evaluation of the Mortality in Emergency Department Sepsis score combined with procalcitonin in septic patients. Am J Emerg Med. 2013;31(7):1086-91.

20. Henning DJ, Bhatraju PK, Johnson NJ, Kosamo S, Shapiro NI, Zelnick LR, et al. Physician Judgment and Circulating Biomarkers Predict 28-Day Mortality in Emergency Department Patients. Crit Care Med. 2019;47(11):1513-21.

21. Levy MM, Fink MP, Marshall JC, Abraham E, Angus D, Cook D, et al. 2001 SCCM/ESICM/ACCP/ATS/SIS International Sepsis Definitions Conference. Crit Care Med. 2003;31(4):1250-6.

22. Zhang Q, Dong G, Zhao X, Li CS. High immunoglobulin E values at admission predict mortality in ED patients with sepsis. Am J Emerg Med. 2016;34(8):1589-94.

23. Wang M, Zhang Q, Zhao X, Dong G, Li C. Diagnostic and prognostic value of neutrophil gelatinase-associated lipocalin, matrix metalloproteinase-9, and tissue inhibitor of matrix metalloproteinases-1 for sepsis in the Emergency Department: an observational study. Crit Care. 2014;18(6):634.

24. Zhang Q, Dong G, Zhao X, Wang M, Li CS. Prognostic significance of hypothalamic-pituitary-adrenal axis hormones in early sepsis: a study performed in the emergency department. Intensive Care Med. 2014;40(10):1499-508.

25. Chen YX, Li CS. Arterial lactate improves the prognostic performance of severity score systems in septic patients in the ED. Am J Emerg Med. 2014;32(9):982-6.

26. Liu B, Chen YX, Yin Q, Zhao YZ, Li CS. Diagnostic value and prognostic evaluation of Presepsin for sepsis in an emergency department. Crit Care. 2013;17(5):R244.

27. Nino ME, Serrano SE, Nino DC, McCosham DM, Cardenas ME, Villareal VP, et al. TIMP1 and MMP9 are predictors of mortality in septic patients in the emergency department and intensive care unit unlike MMP9/TIMP1 ratio: Multivariate model. PLoS One. 2017;12(2):e0171191.

28. Yamamoto S, Yamazaki S, Shimizu T, Takeshima T, Fukuma S, Yamamoto Y, et al. Prognostic utility of serum CRP levels in combination with CURB-65 in patients with clinically suspected sepsis: a decision curve analysis. BMJ Open. 2015;5(4):e007049.

29. Yu H, Nie L, Liu A, Wu K, Hsein YC, Yen DW, et al. Combining procalcitonin with the qSOFA and sepsis mortality prediction. Medicine (Baltimore). 2019;98(23):e15981.

30. Song J, Park DW, Moon S, Cho HJ, Park JH, Seok H, et al. Diagnostic and prognostic value of interleukin-6, pentraxin 3, and procalcitonin levels among sepsis and septic shock patients: a prospective controlled study according to the Sepsis-3 definitions. BMC Infect Dis. 2019;19(1):968.

31. Zhao Y, Jia Y, Li C, Fang Y, Shao R. The risk stratification and prognostic evaluation of soluble programmed death-1 on patients with sepsis in emergency department. Am J Emerg Med. 2018;36(1):43-8.

32. Ranganathan P, Pramesh CS, Aggarwal R. Common pitfalls in statistical analysis: Logistic regression. Perspect Clin Res. 2017;8(3):148-51. 
33. Peduzzi P, Concato J, Kemper E, Holford TR, Feinstein AR. A simulation study of the number of events per variable in logistic regression analysis. J Clin Epidemiol. 1996;49(12):1373-9.

34. Spittler A, Razenberger M, Kupper H, Kaul M, Hackl W, Boltz-Nitulescu G, et al. Relationship between interleukin-6 plasma concentration in patients with sepsis, monocyte phenotype, monocyte phagocytic properties, and cytokine production. Clin Infect Dis. 2000;31(6):1338-42.

35. Ma L, Zhang H, Yin YL, Guo WZ, Ma YQ, Wang YB, et al. Role of interleukin-6 to differentiate sepsis from non-infectious systemic inflammatory response syndrome. Cytokine. 2016;88:126-35.

36. Schultz M, Rasmussen LJH, Andersen MH, Stefansson JS, Falkentoft AC, Alstrup M, et al. Use of the prognostic biomarker suPAR in the emergency department improves risk stratification but has no effect on mortality: a clusterrandomized clinical trial (TRIAGE III). Scand J Trauma Resusc Emerg Med. 2018;26(1):69.

37. Huang Q, Xiong H, Yan P, Shuai T, Liu J, Zhu L, et al. The Diagnostic and Prognostic Value of suPAR in Patients with Sepsis: A Systematic Review and Meta-Analysis. Shock. 2020;53(4):416-25.

38. Vijayan AL, Vanimaya, Ravindran S, Saikant R, Lakshmi S, Kartik R, et al. Procalcitonin: a promising diagnostic marker for sepsis and antibiotic therapy. J Intensive Care. 2017;5:51.

39. van der Does Y, Limper M, Jie KE, Schuit SCE, Jansen H, Pernot N, et al. Procalcitonin-guided antibiotic therapy in patients with fever in a general emergency department population: a multicentre non-inferiority randomized clinical trial (HiTEMP study). Clin Microbiol Infect. 2018;24(12):1282-9.

40. Sankoff JD, Goyal M, Gaieski DF, Deitch K, Davis CB, Sabel AL, et al. Validation of the Mortality in Emergency Department Sepsis (MEDS) score in patients with the systemic inflammatory response syndrome (SIRS). Crit Care Med. 2008;36(2):421-6.

41. Vafaei A, Heydari K, Hashemi-Nazari SS, Izadi N, Hassan Zadeh H. PIRO, SOFA and MEDS Scores in Predicting OneMonth Mortality of Sepsis Patients; a Diagnostic Accuracy Study. Arch Acad Emerg Med. 2019;7(1):e59.

42. Badrinath K, Shekhar M, Sreelakshmi M, Srinivasan M, Thunga G, Nair S, et al. Comparison of Various Severity Assessment Scoring Systems in Patients with Sepsis in a Tertiary Care Teaching Hospital. Indian J Crit Care Med. 2018;22(12):842-5.

43. Sam S, Corbridge TC, Mokhlesi B, Comellas AP, Molitch ME. Cortisol levels and mortality in severe sepsis. Clin Endocrinol (Oxf). 2004;60(1):29-35.

44. Mellhammar L, Linder A, Tverring J, Christensson B, Boyd JH, Sendi P, et al. NEWS2 is Superior to qSOFA in Detecting Sepsis with Organ Dysfunction in the Emergency Department. J Clin Med. 2019;8(8).

\section{Appendix}

\section{Appenix A}

\section{Complete search strategy}

\section{embase.com}

('sepsis'/exp OR 'infection'/exp OR pneumonia/exp OR (sepsis OR septic* OR Bacteremia OR Bacteraemia OR fungemia OR fungaemia OR urosepsis OR infection* OR pneumonia* OR meningitis* OR meningoencephalitis*):ab,ti) AND ('emergency ward'/exp OR 'emergency patient'/de OR 'emergency health service'/de OR 'hospital emergency service'/de OR 'emergency care'/de OR 'emergency physician'/de OR 'emergency medicine'/de OR (((emergency) NEAR/6 (ward* OR department* OR room OR unit OR cent* OR patient* OR care OR healthcare OR physician^ OR medicine)) OR 'acute care' OR ed):ab,ti) AND ('biological marker'/exp OR 'marker'/de OR 'molecular marker'/de OR 'disease marker'/de OR 'blood level'/de OR 'lactate blood level'/de OR 'protein blood level'/de OR ((biological* OR bio* OR inflammat* OR diagnos* OR molecul* OR disease) NEAR/3 marker*) OR biomarker* OR ((serum OR blood) NEAR/6 (concentrate* OR level* OR marker* OR lactate OR protein* 
OR elevat*))):ab,ti) NOT ('case report'/de OR (case-report*):ti) NOT ([Conference Abstract]/lim) AND [English]/lim NOT ((juvenile/exp OR pediatrics/exp OR 'newborn sepsis'/de OR 'pediatric emergency medicine'/de OR (child* OR adolescen* OR infan* OR newborn* OR neonat*):ti) NOT (adult/exp OR (adult*):ti)) NOT ([animals]/lim NOT [humans]/lim)

\section{Medline Ovid}

(exp Sepsis/ OR exp Infection/ OR exp Pneumonia/ OR (sepsis OR septic* OR Bacteremia OR Bacteraemia OR fungemia OR fungaemia OR urosepsis OR infection* OR pneumonia* OR meningitis* OR meningoencephalitis*).ab,ti.) AND (Emergency Medical Services/ OR Emergency Service, Hospital/ OR Emergency Medicine/ OR (((emergency) ADJ6 (ward* OR department* OR room OR unit OR cent* OR patient* OR care OR healthcare OR physician* OR medicine)) OR acute care OR ed).ab,ti.) AND (exp Biomarkers/ OR blood.fs. OR (((biological* OR bio* OR inflammat* OR diagnos* OR molecul* OR disease) ADJ3 marker*) OR biomarker* OR ((serum OR blood) ADJ6 (concentrate* OR level* OR marker* OR lactate OR protein* OR elevat*))).ab,ti.) NOT (case reports/ OR (case-report*).ti.) AND english.la. NOT ((exp child/ OR exp infant/ OR Pediatrics/ OR Neonatal Sepsis/ OR Pediatric Emergency Medicine/ OR (child* OR adolescen* OR infan* OR newborn* OR neonat*).ti.) NOT (exp adult/ OR (adult*).ti.)) NOT (exp animals/ NOT humans/)

\section{Web of science}

TS=(((sepsis OR septic* OR Bacteremia OR Bacteraemia OR fungemia OR fungaemia OR urosepsis OR infection* OR pneumonia* OR meningitis* OR meningoencephalitis*)) AND ((((emergency) NEAR/5 (ward* OR department* OR room OR unit OR cent* OR patient* OR care OR healthcare OR physician* OR medicine)) OR "acute care" OR ed)) AND ((((biological* OR bio* OR inflammat* OR diagnos* OR molecul* OR disease) NEAR/2 marker*) OR biomarker* OR ((serum OR blood) NEAR/5 (concentrate* OR level* OR marker* OR lactate OR protein* OR elevat*) $)))$ ) NOT TI=(((child* OR adolescen* OR infan* ORnewborn* OR neonat*)) NOT ((adult*))) AND DT=(article) AND LA=(english)

\section{Cochrane CENTRAL}

((sepsis OR septic* OR Bacteremia OR Bacteraemia OR fungemia OR fungaemia OR urosepsis OR infection* OR pneumonia* OR meningitis* OR meningoencephalitis*):ab,ti) AND ((((emergency) NEAR/6 (ward* OR department* OR room OR unit OR cent* OR patient* OR care OR healthcare OR physician* OR medicine)) OR 'acute care' OR ed):ab,ti) AND (((biological* OR bio* OR inflammat* OR diagnos* OR molecul* OR disease) NEAR/3 marker*) OR biomarker* OR ((serum OR blood) NEAR/6 (concentrate* OR level* OR marker* OR lactate OR protein* OR elevat*))):ab,ti) NOT (((child* OR adolescen* OR infan* OR newborn* OR neonat*):ti) NOT ((adult*):ti))

\section{Google scholar}

sepsis|septic|Bacteremia|Bacteraemia|fungemia|fungaemia|urosepsis|infection|pneumonia|meningitis|meningoencephalitis "emergency ward|department|room"|"acute care" "biological|bio|inflammatory marker|markers"|biomarker|biomarkers -child infant -newborn

\section{Figures}




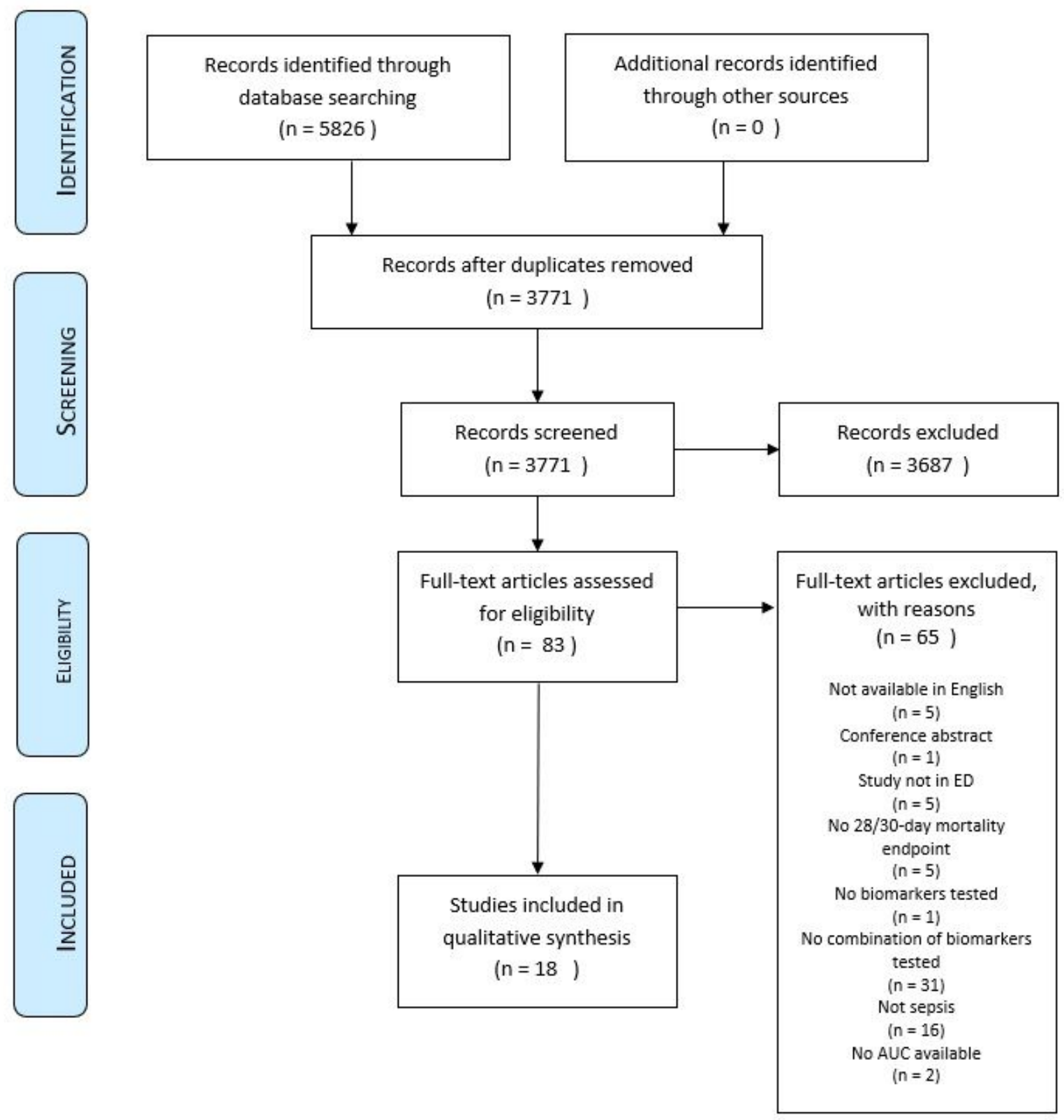

Figure 1

Flow chart of included articles 